\title{
Real-Time Evaluation of Compaction Quality by Using Artificial Neural Networks
}

\author{
Weidong Cao, ${ }^{1}$ Shutang Liu, ${ }^{1}$ Xuechi Gao, ${ }^{2}$ Fei Ren $\left(\mathbb{D},{ }^{3}\right.$ Peng Liu, ${ }^{2}$ and Qilun Wu ${ }^{1}$ \\ ${ }^{1}$ School of Qilu Transportation, Shandong University, Jinan 250061, Shandong Province, China \\ ${ }^{2}$ Shandong Hi-Speed Group Co. Ltd., Jinan 250101, Shandong Province, China \\ ${ }^{3}$ School of Mechanical and Automotive Engineering, Qilu University of Technology (Shandong Academy of Sciences), No. 3501, \\ Daxue Road, Changqing District, Jinan 250353, Shandong Province, China \\ Correspondence should be addressed to Fei Ren; ren87@outlook.com
}

Received 27 October 2020; Revised 9 December 2020; Accepted 13 December 2020; Published 23 December 2020

Academic Editor: Yubo Jiao

Copyright (c) 2020 Weidong Cao et al. This is an open access article distributed under the Creative Commons Attribution License, which permits unrestricted use, distribution, and reproduction in any medium, provided the original work is properly cited.

\begin{abstract}
The primary goal of this study is to find an easy and convenient way to estimate the degree of compaction in real time for compaction quality control. In this paper, an artificial neural network classifier is developed to identify the different characteristic patterns of drum vibration and classify them according to the different compaction levels. At first, a field compaction experiment is designed and performed in a construction site, and the degree of compaction and the vibration are measured. Then, the vibration signals collected from the experiment are processed to extract the features of vibration patterns and labeled with the compaction level to train the artificial neural network model. At last, the performance of the artificial neural network classifier is verified against the degree of compaction measured by using a nuclear density gauge. It can be found that artificial neural networks show good performance and huge potential for the problem of compaction quality control.
\end{abstract}

\section{Introduction}

The compaction process plays an important role in improving the strength and bearing capacity of materials for use in road construction. The existing compaction quality control relies on spot tests, such as sand replacement method, falling weight deflectometer (FWD), and plate bearing test. These traditional manual measurements have several drawbacks [1,2]: (1) the measurements are usually time consuming and may interrupt the subsequent construction operation; (2) test samples are collected at limited test points, and the testing results cannot indicate the overall pavement quality; (3) the measurements are performed after compaction; thus, it is impossible to provide real-time compaction quality information for the operator, which may lead to under or over compaction. To address these problems, intelligent compaction (IC) technique is proposed to provide real-time compaction quality assurance during compaction.
So far, there are several equipment manufacturers around the world offering IC rollers to compact subgrade and aggregate materials. Several intelligent compaction measurement values (ICMV) are set up to evaluate the compaction quality, such as Compaction Meter Value (CMV), Compaction Control Value (CCV), Resonance Meter Value (RMV), Machine Drive Power (MDP), vibration modulus (Evib), and soil stiffness (Ks) $[2,3]$. CMV is widely accepted for quality assurance, and it is computed by the amplitude of vertical drum acceleration at the operating frequency and first harmonic. CCV and RMV further consider the high-order harmonics. Considering the nonlinearity vibration induced by the periodic loss of contact between soil and drum, Anderegg et al. $[4,5]$ develop a feedback control system to automatically adjust the compaction parameters (vibration frequency, vibration amplitude, and driving speed) during construction. Due to the development of these helpful IC technologies, roller operator can optimize the compaction process timely according to the 
updated compaction information, and the compaction quality is improved effectively.

However, recent research studies found that there are still some uncertain correlations between ICMVs and compaction quality. Firstly, CMV, as a harmonic-based indicator, is easily influenced by many factors. Zhu et al. [6] test a multilayer structure and find that CMV is sensitive to the characteristic of underlying layers, such as stiffness and moisture content of the layers. White et al. [7] indicate that CMV is dependent on the vibration amplitude; therefore, a higher excitation force amplitude generally yields a greater CMV at a constant soil modulus. Wersäll et al. [8] conduct full-scale tests to study the influence of variable frequencies on compaction control. The results indicate that the resonant frequency is about $17 \mathrm{~Hz}$, and the optimum compaction frequency is about $18 \mathrm{~Hz}$, while the standard operating frequency of the roller is about $31 \mathrm{~Hz}$. This means that there is no direct correlation between the excitation force and compaction quality. Secondly, the mechanical-based ICMVs related to soil stiffness and vibration modulus manifest unstable changes due to their amplitude dependence. Mooney and Rinehart $[9,10]$ demonstrate that soil stiffness on the soft layer decreases with increasing excitation force, while on the stiffer layer exhibits conversely. Further analysis by Mazari et al. [11] shows that roller type, machine operation setting variation, and instability of the machine in practicing operation commonly affect the accuracy of ICMVs. Furthermore, some researchers [12-16] use operational modal analysis (OMA) for the structural health monitoring of engineering. Different from the experimental modal analysis methods, OMA uses the output-only response to identify the structure properties; thus, the input excitation measures can be avoided.

Recently, an artificial intelligence-based intelligent compaction analyzer (ICA) was developed by Barman et al. [17-19]. The frequency characteristics of drum vibration can be analyzed by the ICA, and amounts of field testing show that the results correlate well with subgrade modulus. Zhang et al. [20, 21] utilize acoustic wave detection techniques to evaluate rock-fill compaction status, and a genetic algorithm-based optimization procedure was proposed to optimize the overall compaction process.

The recent IC techniques are largely based on the vibration analysis of pavement during compaction, trying to build the correlation between the vibration of pavement and compaction quality. However, the development of IC technique is blocked by the complication of vibration during compaction. From another point of view, the development of IC technique can be considered as a problem of signal processing and recognition. Fourier transform and artificial neural networks (ANN) have been widely used for signal processing and recognition nowadays, especially for speech recognition [22-25]. Zhan et al. [26] also use the ANN for radar waveform recognition. These studies provide many references on the applications of Fourier transform and ANN. However, few studies have been conducted on the compaction quality control by using the ANN. In the research studies by Barman et al. [17-19], the ANN is tried to analyze the correlation between the vibration pattern and subgrade stiffness. For the development of IC technique, neural networks can bypass some difficulties which cannot be solved easily by traditional methods, showing huge potential.

Generally, degree of compaction is the most direct index for the compaction quality evaluation. The main objective of this study is to find an easy and convenient way to estimate the degree of compaction in real time. In this research, the compaction analysis is based on a hypothesis that the vibratory roller and pavement form a coupled system during compaction. The coupled response is determined by the excitation frequency and natural vibration modes of the coupled system. The variations in the degree of compaction will affect the response and will lead to different patterns of vibrations of the drum. Therefore, the compaction quality can be estimated by using the mapping between the vibration pattern and degree of compaction.

In reality, however, the vibration pattern of the drum usually includes the information of the noise, which means some of the features of the vibration pattern reflect the system and others reflect the noise. The vibration pattern features reflecting the system can be used to estimate the compaction quality, but these useful features cannot be recognized and extracted easily. In this paper, an artificial neural network (ANN) classifier is developed to identify the different characteristic patterns of drum vibration and classify them according to the different compaction levels. A field compaction experiment is designed and performed in a construction site, and the degree of compaction and the vibration are measured. The vibration signals collected from the experiment are processed to extract the features of vibration patterns and then labeled with the compaction level to train the ANN model. At last, the performance of the ANN classifier is verified against the degree of compaction measured by using a nuclear density gauge (NDG).

\section{Experimental Program and Signal Processing}

It is assumed that the vibratory roller and pavement underneath form a coupled system during compaction. The variations in degree of compaction affect the coupled response and lead to different vibration patterns of the drum. To analyze and make use of the mapping relationship between the vibration pattern and degree of compaction, a field compaction experiment is designed and performed in a construction site to collect the data of the vibration signal and degree of compaction.

2.1. Experimental Program. A field test is performed on the extension project of G2 expressway in Shandong Province, China. The typical pavement structure used in the project is shown in Table 1, which consists of three hot-mix asphalt (HMA) surface layers, one flexible base layer, two semirigid base layers, one sub-base layer, and the subgrade, in that order, from top to bottom. The vibrating compaction test is implemented at the cement-stabilized gravel base layer with a thickness of $18 \mathrm{~cm}$. The aggregate used for cementstabilized base layer is limestone, and the details of the mixture and equipment are shown in Tables 2 and 3. 
TABle 1: Pavement structure in the project.

\begin{tabular}{lc}
\hline & $4 \mathrm{~cm}$ SMA-13 \\
HMA surface layers & $6 \mathrm{~cm} \mathrm{AC-20}$ \\
& $8 \mathrm{~cm}$ AC- 25 \\
Flexible base layer & $12 \mathrm{~cm}$ LSPM-25 \\
Semirigid base layer & $2 \times 18 \mathrm{~cm}$ cement-stabilized aggregate \\
Sub-base layer & $18 \mathrm{~cm}$ cement-stabilized weathered sands \\
Subgrade & Compacted natural soil \\
\hline
\end{tabular}

TABLe 2: Details of the base layer.

\begin{tabular}{cccccc}
\hline Layer & Materials & Depth $(\mathrm{cm})$ & Cement mass percent $(\%)$ & Maximum dry density $\left(\mathrm{g} / \mathrm{cm}^{3}\right)$ & $\begin{array}{c}\text { Optimum moisture content } \\
(\%)\end{array}$ \\
\hline $\begin{array}{c}\text { Base } \\
\text { Cement-stabilized } \\
\text { gravel }\end{array}$ & $18 \times 2$ & 4.5 & 2.348 & 4.9 \\
\hline
\end{tabular}

TABLE 3: Details of equipment.

\begin{tabular}{lccccccc}
\hline $\begin{array}{l}\text { Roller } \\
\text { type }\end{array}$ & $\begin{array}{c}\text { Drum width } \\
(\mathrm{m})\end{array}$ & $\begin{array}{c}\text { Static weight } \\
(\mathrm{kg})\end{array}$ & $\begin{array}{c}\text { Speed } \\
(\mathrm{m} / \mathrm{s})\end{array}$ & $\begin{array}{c}\text { Operation frequency } \\
(\mathrm{Hz})\end{array}$ & $\begin{array}{c}\text { Amplitude } \\
(\mathrm{mm})\end{array}$ & $\begin{array}{c}\text { Excitation force } \\
(\mathrm{KN})\end{array}$ & Sample frequency \\
\hline $\begin{array}{l}\text { Single } \\
\text { drum }\end{array}$ & 2.13 & 2000 & $0.5 \sim 0.7$ & 33 & 1.2 & 290 & $2000 \mathrm{~Hz}$ \\
\end{tabular}

Two wireless accelerometers are mounted on the axle of the roller drum on both sides to monitor the vibration acceleration signals in the vertical direction, as shown in Figure 1. The compaction is carried out on ten test lanes, and each test lane has a length of $60 \mathrm{~m}$. The width of each test lane is $2.13 \mathrm{~m}$, the same as the width of the roller. Figure 2 shows the roller pass trajectory on each test lane, and a total of 8 passes are performed for each lane. The degree of compaction is measured by using nuclear density gauge for each pass and computed as follows:

$$
\begin{aligned}
\rho_{d} & =\frac{\rho_{w}}{1+w}, \\
\text { DOC } & =\frac{\rho_{d}}{\rho_{E}} \times 100 \%,
\end{aligned}
$$

where DOC is the degree of compaction, $\rho_{E}$ is maximum dry density, $\rho_{w}$ and $w$ are wet density and moisture content measured by nuclear density gauge, and $\rho_{d}$ is dry density. For each test lane, two different locations are tested, as shown in Figure 2, and the relationship between the number of roller passes and degree of compaction is investigated.

The relationship between the number of passes and degree of compaction is plotted in Figure 3. Here, the open circles denote the degree of compaction obtained from the experiment by using NDG, and the solid circles denote the average degree of compaction for each pass. Ten test lanes are investigated and each test lane has two test locations; therefore, there are 20 experimental results for each pass. For the cement-stabilized gravel base, the minimum requirement of degree of compaction is $98 \%$ and about 6 passes are needed to reach the requirement.

2.2. Signal Processing. The vibration signals are collected continuously from two accelerometers when the vibratory roller moves on the test lane. The length of the test lane is
$60 \mathrm{~m}$, the velocity of the roller is about $0.6 \mathrm{~m} / \mathrm{s}$, and it takes about $100 \mathrm{~s}$ for one pass. Therefore, we can get a 100 -second long vibration signal from one accelerometer in one pass, and a total of 160 long signals can be collected since we have ten test lanes and two accelerometers. Theoretically, the vibration signals from two accelerometers have the same frequency components, even though their amplitudes may be different.

The vibration is sampled at a $2 \mathrm{kHz}$ sampling frequency. The long vibration signal of one pass is divided into lots of 0.5 -second short signals. Each short signal includes 1000 contiguous data samples having an overlap with 500 previous values. Each short signal is converted to frequency domain representation using a fast Fourier transform (FFT). Since the vibration is sampled at $2 \mathrm{kHz}$, the Nyquist frequency is $1 \mathrm{kHz}$. Therefore, a single-sided FFT provided a frequency spectrum distributed between 0 and $1 \mathrm{kHz}$, and the output of the single-sided FFT for each short signal is an array of 500 elements, expressed as $a=\left(a_{1}, a_{2}, \ldots, a_{500}\right)$. By using a FFT, the features of the vibration signal are expressed as the frequency components. Amplitude is not considered as a kind of feature in this study; array $a$ should be normalized to eliminate the effects of amplitudes. The normalized array $x$ is obtained as follows:

$$
\begin{aligned}
& \bar{a}=\ln (a), \\
& x=\frac{\bar{a}}{|\bar{a}|_{\max }} .
\end{aligned}
$$

Here, the logarithmic operation in equation (2) is used to amplify some inconspicuous frequency components. The signal processing method in this section is shown in Figure 4 and summarized as the following steps: 


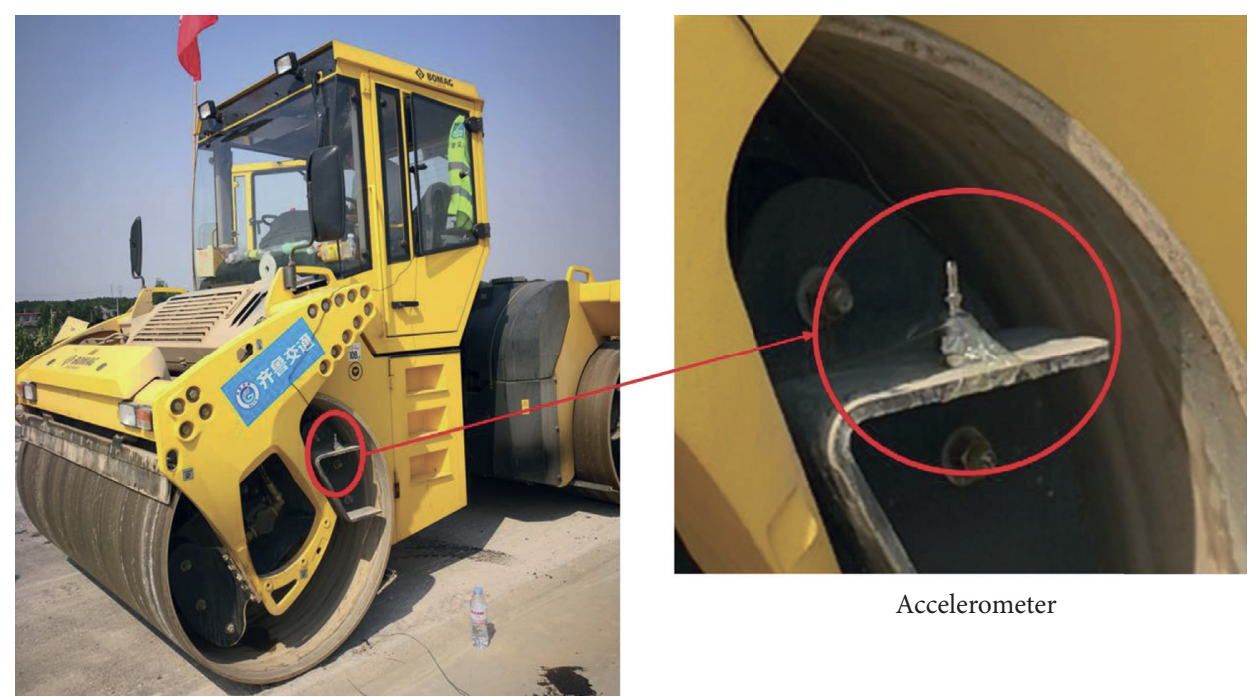

FIGURE 1: Accelerometer-equipped vibratory roller during compaction.

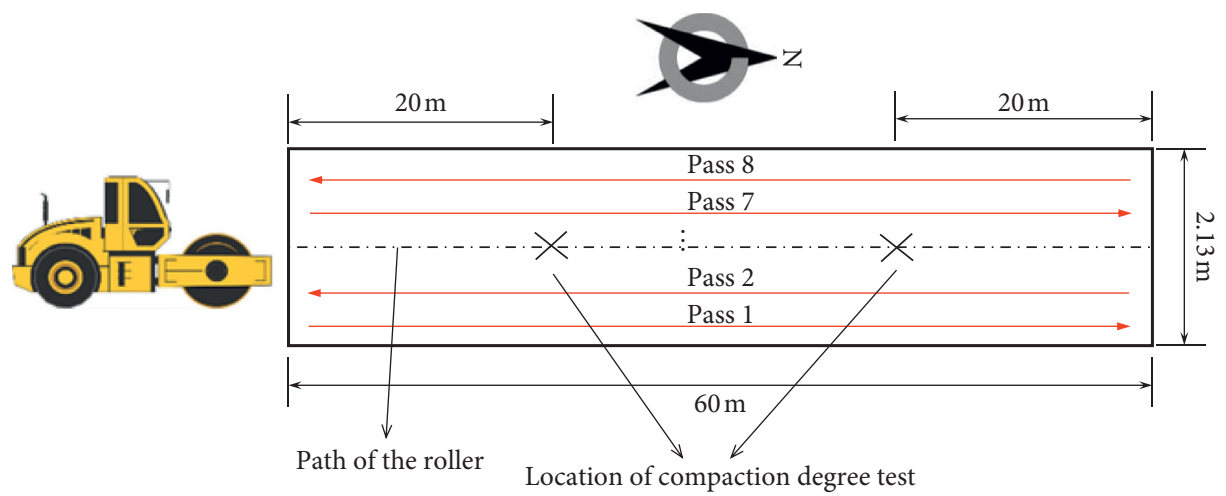

Figure 2: Test lane.

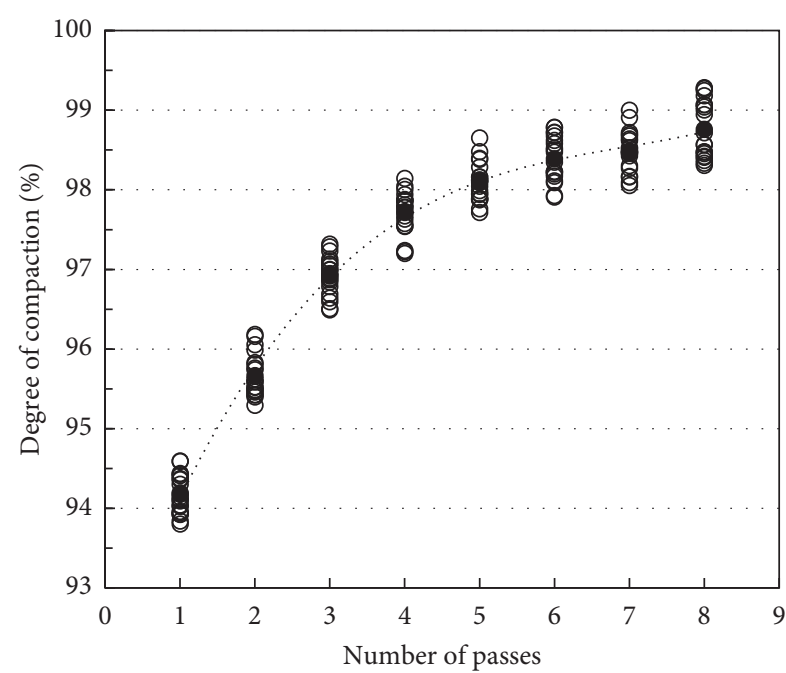

○ Experimental results

- Average value for each pass

FIGURE 3: Influence of number of passes on the degree of compaction.
(1) The long vibration signal from the accelerometer in one pass is divided into lots of 0.5 -second short signals

(2) Each short signal is converted to frequency domain representation by using a single-sided FFT to extract the frequency features

(3) The output of the single-sided FFT for each short signal is normalized by using equation (2) to eliminate the effects of amplitudes and amplify the inconspicuous frequency components

(4) The processed signals are input to ANN for training or for predicting the degree of compaction

The frequency features of vibration signals are extracted by this method, and the processed arrays will be used as input data for training the ANN model. In the following discussion on the ANN, the input array $x$ is called "sample," and the element of $x$ is called "feature." In this study, therefore, each sample has 500 features.

Before being used for training the ANN, each sample should be labeled with a target class. In this research, we use four target classes to represent four compaction levels, as shown in Table 4. According to the experimental results of 


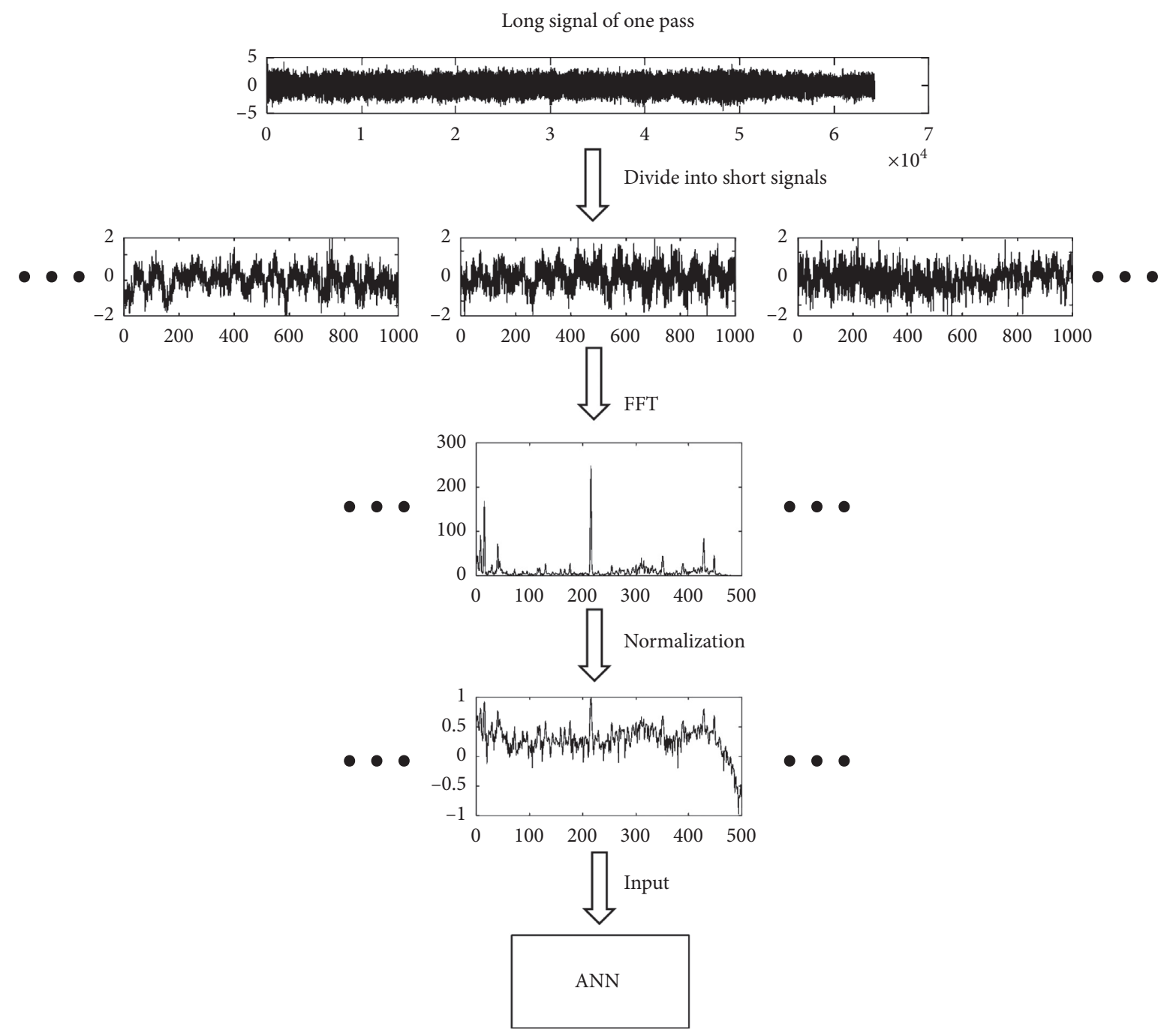

Figure 4: Signal processing.

TABLE 4: Target class and compaction level.

\begin{tabular}{lccc}
\hline & Level no. & Degree of compaction (\%) & Target array \\
\hline Target class 1 & 1 & $<94$ & $(1,0,0,0)$ \\
Target class 2 & 2 & $94 \sim 96$ & $(0,1,0,0)$ \\
Target class 3 & 3 & $96 \sim 98$ & $(0,0,1,0)$ \\
Target class 4 & 4 & $>98$ & $(0,0,0,1)$ \\
\hline
\end{tabular}

the degree of compaction (Figure 3), the samples are labeled with the corresponding compaction level, forming the dataset for the ANN training.

\section{Development of the ANN Model}

A multilayer perceptron (MLP) feedforward neural network is used in this research. Figure 5 shows the structure of the network. The network consists of one input layer, two hidden layers, and an output layer. There are 500 nodes in the input layer since each sample has 500 features. The first and second hidden layers contain 44 and 10 nodes, respectively. The output layer contains four nodes representing four classes of the compaction level. Figure 5(b) shows the schematic of a single neuron. Each node is governed by the following equation:

$$
x_{s, j}^{(l+1)}=f\left(\sum_{i=1}^{k} x_{s, i}^{(l)} w_{i, j}^{(l)}+b_{0, j}^{(l)}\right),
$$

where the subscript $s$ denotes the sth sample, $k$ denotes the number of nodes in layer $l, x_{s, i}^{(l)}$ is the $i$ th input of layer $l$, and $x_{s, j}^{(l+1)}$ is the $j$ th output. Meanwhile, $x_{s, j}^{(l+1)}$ is also the $j$ th input of layer $l+1 . w_{i, j}^{(l)}$ is the weight value from the $i$ th input to the $j$ th output. $b_{0, j}^{(l)}$ is the weight value from the bias term of layer $l$ to the jth output. All the bias terms are " +1 " in this research. $f()$ is the activation function, a softmax function, $\operatorname{Sof}\left(z_{j}\right)=$ $\left(e^{z_{j}} / \sum_{i=1}^{4} e^{z_{i}}\right)$, is used in the output layer to ensure the predictions for each sample are in the range of $[0,1]$ and sum to 1 , and a sigmoid function, $\operatorname{Sig}(z)=\left(1+e^{-z}\right)^{-1}$, is used in the rest of the layers.

\section{Training of the ANN Model}

A supervised learning method is used to train the ANN. The network can be trained to classify inputs according to target 


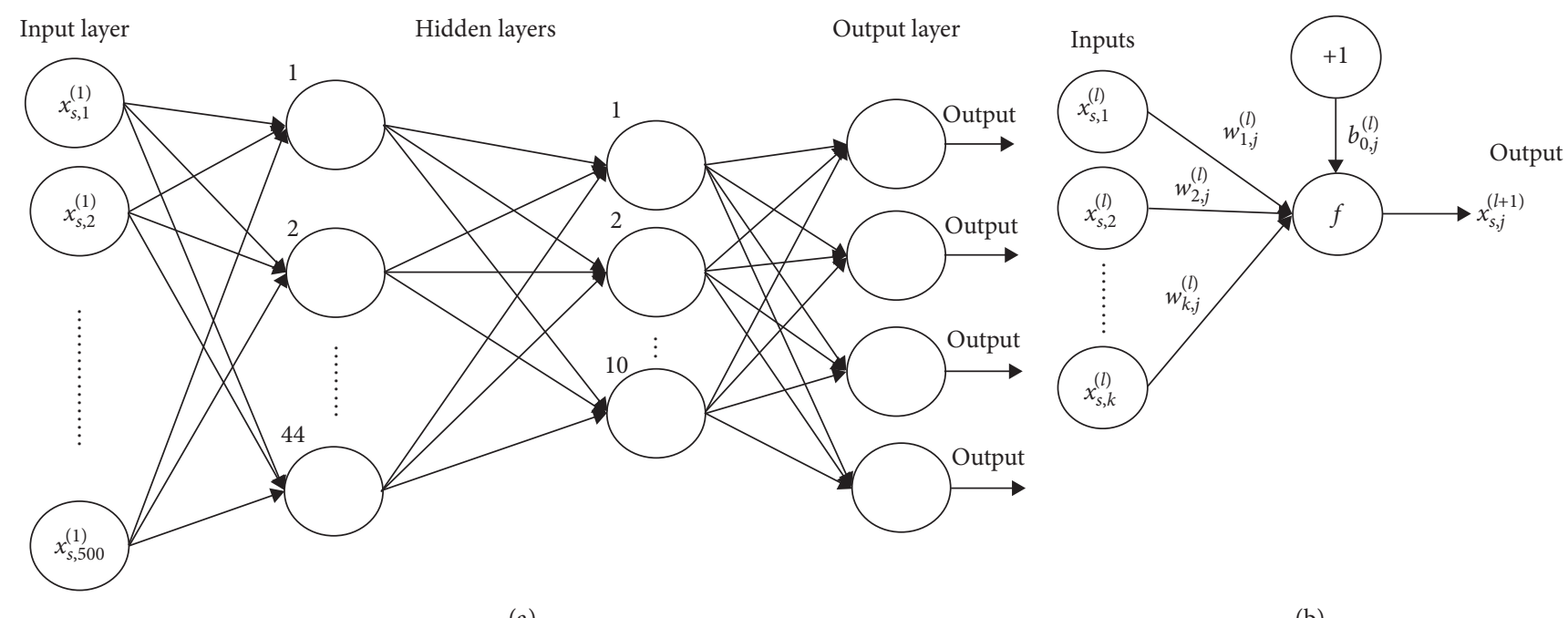

Figure 5: (a) Structure of the MLP feedforward ANN and (b) schematic of a neuron.

classes. The target data should consist of arrays of all 0 values except for a 1 in element $c$, where $c$ is the class they are to represent, as shown in Table 4.

The cross-entropy is used as the loss function to measure the network's performance. The loss associated with the sth prediction would be

$$
\mathrm{CE}_{s}=-\sum_{c=1}^{4} y_{s, c} \ln \left(\widehat{y}_{s, c}\right),
$$

where $y$ is the target array, $\hat{y}$ is the output array of the output layer, the subscript $s$ denotes the sth sample, and $c$ denotes the $c$ th element of the target or output array. The crossentropy loss of the entire training dataset would be the average $\mathrm{CE}_{s}$ over all samples.

In this work, the scaled conjugate gradient (SCG) algorithm [27] is used to perform training. SCG is based on a class of optimization algorithms called Conjugate Gradient Methods (CGM), but this algorithm avoids the line-search per learning iteration by using a Levenberg-Marquardt approach to scale the step size. SCG can train any network as long as its weight, net input, and activation functions have derivative functions. Backpropagation is used to calculate derivatives of the loss function with respect to the weights.

To avoid overfitting during neural network training, the dataset is randomly divided into three subsets: training set, validation set, and test set. The training set is used for computing the gradient and updating the network weights. The validation set is aimed to avoid the overfitting problem. The error on the validation set normally decreases during the initial phase of the training, as does the error on the training set. When the network begins to overfit the data, the validation error typically begins to increase. When the validation error increases for several iterations, the training should be stopped. In this work, when the validation error keeps increasing for six iterations, the training is stopped and the weights at the minimum of the validation error are returned.
The test set is not used during the training, but it is used as a completely independent test of network generalization. In this study, validation and test datasets are each set to $15 \%$ of the original data.

The data of the test lanes 1 to 8 are used for the training in this work. The signals collected in these eight test lanes are processed by using the method mentioned in Section 2.2, forming the dataset for the training. The order of samples in the dataset matrix is arranged randomly since samples are considered to be independent of each other. The data of test lane 9 and 10 are used to test the validity and performance of the ANN model.

The training performance of the ANN is shown in Figure 6. The performance is also visualized in the form of confusion matrix in Figure 7. In the confusion matrix in Figure 7, the rows correspond to the output predicted class, and the columns correspond to the target class. The diagonal cells correspond to samples that are correctly classified. The off-diagonal cells correspond to incorrectly classified samples. Both the number of samples and the percentage of the total number of samples are shown in each cell. The column on the far right of the plot shows the percentages of all the samples predicted to belong to each class that are correctly and incorrectly classified. The row at the bottom of the plot shows the percentages of all the samples belonging to each class that are correctly and incorrectly classified. The cell in the bottom right of the plot shows the overall accuracy.

\section{Test Results of the ANN Classifier}

A good training performance of the ANN can be seen in Figure 7. Combining the signal processing method and ANN model, we can get an ANN classifier, as shown in Figure 8. We input a 0.5 -second signal, the ANN classifier outputs an estimated compaction level.

In this section, the data of test lane 9 and 10 are used to test the validity and performance of the ANN classifier. As mentioned in Section 2, a total of 32 long vibration signals 


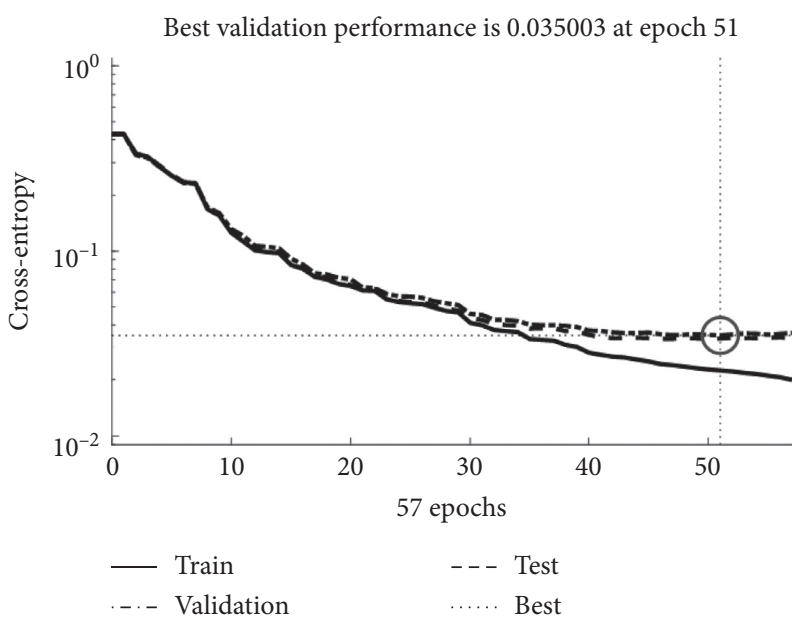

FIGURE 6: Output prediction error of the ANN after each training cycle.

\begin{tabular}{|c|c|c|c|c|c|}
\hline 1 & $\begin{array}{c}799 \\
8.6 \%\end{array}$ & $\begin{array}{c}0 \\
0.0 \%\end{array}$ & $\begin{array}{c}0 \\
0.0 \%\end{array}$ & $\begin{array}{c}0 \\
0.0 \%\end{array}$ & $\begin{array}{c}100 \\
0.0 \%\end{array}$ \\
\hline 2 & $\begin{array}{c}0 \\
0.0 \%\end{array}$ & $\begin{array}{c}2240 \\
24.1 \%\end{array}$ & $\begin{array}{c}55 \\
0.6 \%\end{array}$ & $\begin{array}{c}24 \\
0.3 \%\end{array}$ & $\begin{array}{c}96.6 \% \\
3.4 \%\end{array}$ \\
\hline 3 & $\begin{array}{c}0 \\
0.0 \%\end{array}$ & $\begin{array}{c}57 \\
0.6 \%\end{array}$ & $\begin{array}{c}3237 \\
34.9 \%\end{array}$ & $\begin{array}{c}110 \\
1.2 \%\end{array}$ & $\begin{array}{c}95.1 \% \\
4.9 \%\end{array}$ \\
\hline 4 & $\begin{array}{c}0 \\
0.0 \%\end{array}$ & $\begin{array}{c}19 \\
0.2 \%\end{array}$ & $\begin{array}{c}79 \\
0.9 \%\end{array}$ & $\begin{array}{c}2666 \\
28.7 \%\end{array}$ & $\begin{array}{c}96.5 \% \\
3.5 \%\end{array}$ \\
\hline & $\begin{array}{c}100 \\
0.0 \%\end{array}$ & $\begin{array}{c}96.7 \% \\
3.3 \%\end{array}$ & $\begin{array}{c}96.0 \% \\
4.0 \%\end{array}$ & $\begin{array}{c}95.2 \% \\
4.8 \%\end{array}$ & $\begin{array}{c}96.3 \% \\
3.7 \%\end{array}$ \\
\hline & 1 & 2 & 3 & 4 & \\
\hline
\end{tabular}

(a)

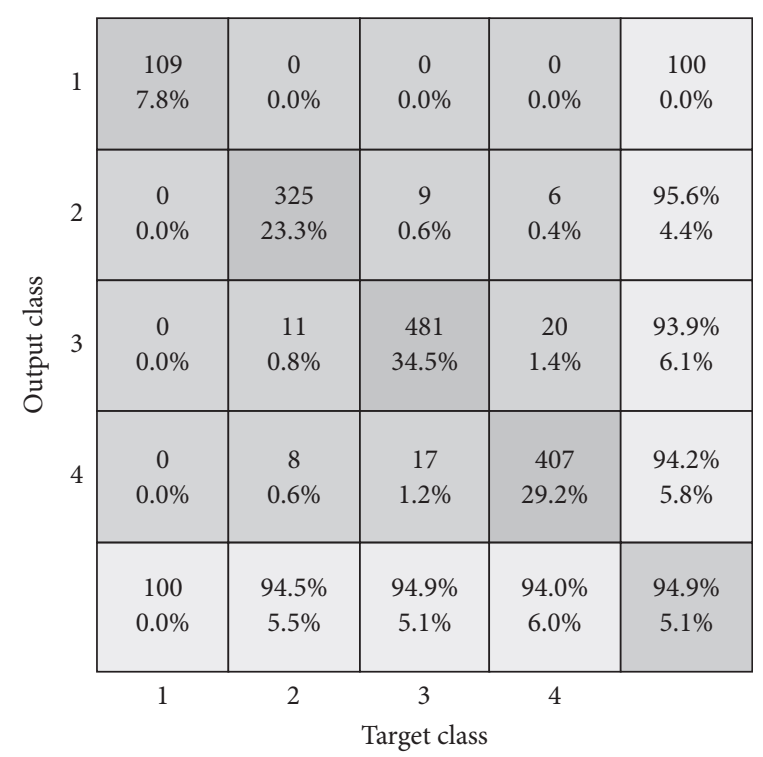

(b)

Figure 7: Confusion matrix of training performance: (a) for all dataset and (b) for test set.

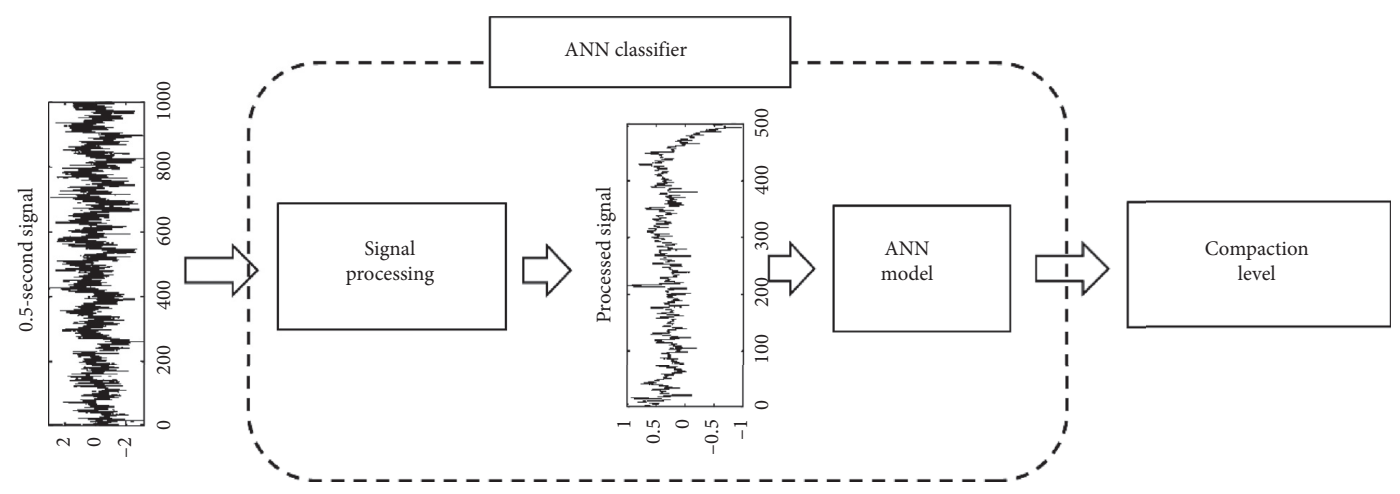

Figure 8: The ANN classifier. 
are collected on these two test lanes. Considering that, under the actual working condition, the signal samples may be not collected continuously or in order during the real-time estimation of the compaction quality, we randomly capture some 0.5 -second short signals from the long signals as the inputs of the ANN classifier. The output accuracy of the ANN classifier is shown in Figure 9. We can believe that the ANN classifier is accurate enough for the real-time estimation of the compaction quality.

\section{Discussion on Roller Moving Direction during Compaction}

The analysis in this paper mainly focuses on the compaction of cement-stabilized gravel base. The main intention of this study is to find an easy way to estimate the degree of compaction in real time. The ANN classifier developed in this paper partly achieves this goal by using vibration pattern recognition. In fact, it is difficult to analyze the vibration of cement-stabilized gravel base layer by using traditional methods because of the inhomogeneity and anisotropy. Therefore, the authors try to use neural networks. Although neural network is a black box for the user, it does work well in this study.

From the experimental results shown in Figure 3, it can be seen that the degree of compaction increases slowly from pass 5 to 8 . In the "sight" of neural network, the vibration signals of pass 5 to 8 should look similar since the values of the degree of compaction have little differences (they are in the same compaction level). In this section, we try to label the samples with the number of passes, and the network is trained to classify the input samples according to 8 target classes. The training performance is shown in Figure 10. Some samples of pass 5 are misclassified as target class 7 , and some samples of pass 6 are misclassified as target class 8 . However, the ANN has good performances on the classification between class 5 and 6 and between class 6 and 7 as well. The ANN thinks some vibration signals of pass 5 look like the signals of pass 7 , but signals of pass 5 and 6 are completely different. This is weird because the values of the degree of compaction of pass 5 and 6 are closer, and signals of pass 5 and 6 should look more similar. Similarly, the ANN thinks some signals of pass 6 look like the signals of pass 8 , but not signals of pass 7 .

The roller moving direction may be the reason for this weird phenomenon. During compaction, in Figure 2, the roller moves to the north in pass $1,3,5$, and 7 , to the south in pass $2,4,6$, and 8 . Due to the inhomogeneity and anisotropy, the cement-stabilized gravel base layer shows different properties and vibration responses in different roller moving directions. The effect of roller moving direction may be paid attention to for the vibration analysis of compaction in future studies.

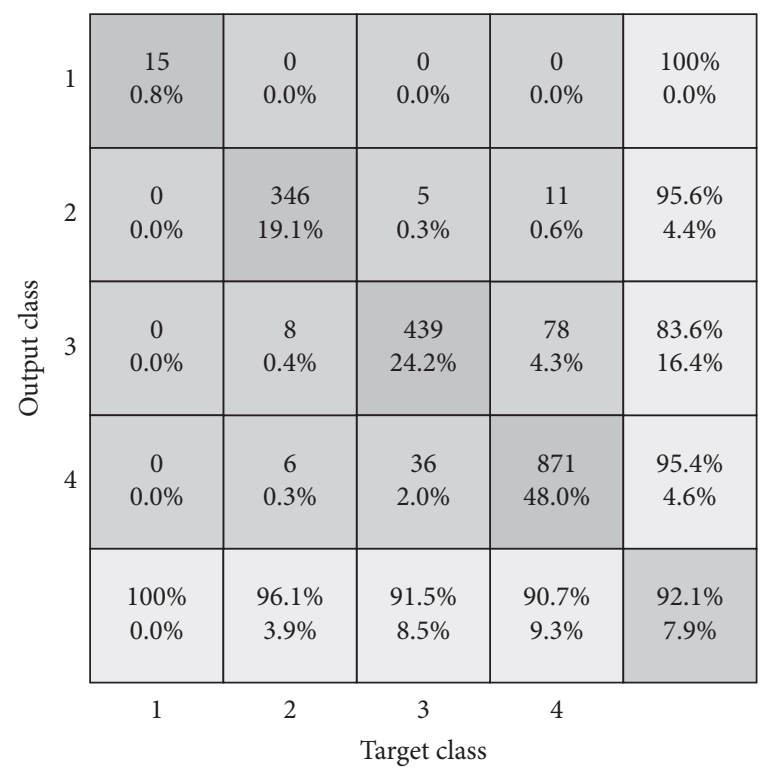

Figure 9: Output accuracy of the ANN classifier.

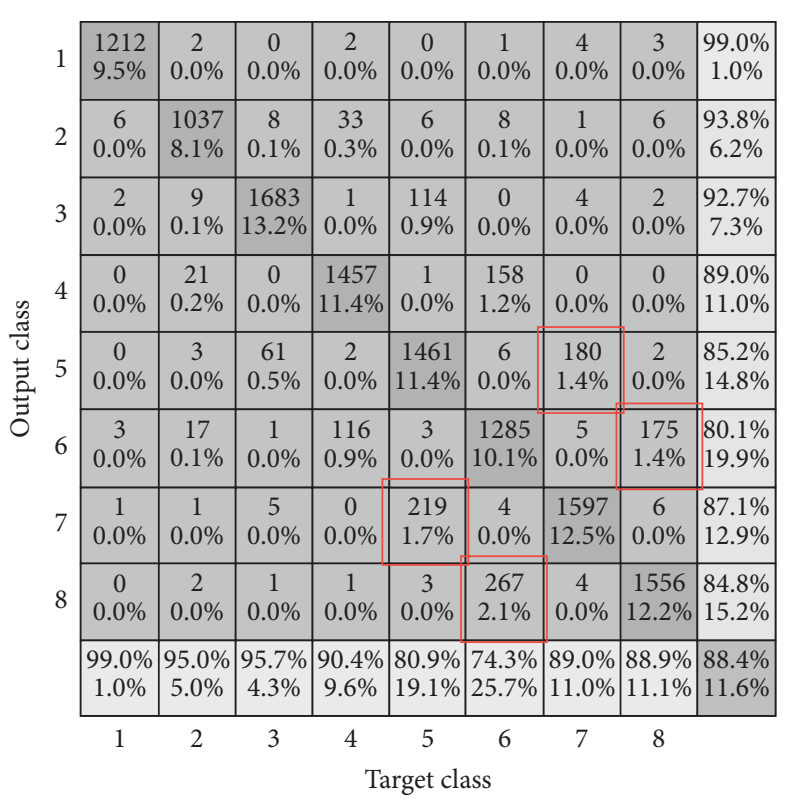

Figure 10: Classification according to the number of passes.

\section{Conclusions and Outlooks}

The primary goal of this paper is to find an easy and convenient way to estimate the degree of compaction in real time. The ANN classifier developed in this paper partly achieves this goal. Some main conclusions and findings of this research are as follows: 
(1) A signal processing method is proposed. The signals collected in the experiment are converted to frequency domain representation by using a singlesided FFT. The frequency features of the vibration signal are extracted and expressed in the logarithmic form to amplify some inconspicuous frequency components. The vibration signals are also normalized to eliminate the effects of amplitudes.

(2) An ANN model is designed and trained to identify the different vibration patterns of drum and classify them according to the different compaction levels. The correlation between vibration patterns and the compaction quality is built by the ANN model.

(3) An ANN classifier is developed by combining the signal processing method and ANN model together. The ANN classifier can estimate the compaction quality in real time according to the input vibration signal. From the testing results, it can be found that the ANN classifier shows a good performance on the compaction quality real-time estimation.

(4) The effect of roller moving direction during compaction is observed and analyzed by using the ANN. This effect may be important and should be considered in future studies.

Essentially, the development of IC technique is based on the idea that the compaction quality can be evaluated by identifying the vibration patterns of pavement. Therefore, IC technique can be regarded as a problem of pattern recognition, and the ANN is very suitable for this problem. However, the works in this paper still have room for improvement. To improve the performance of the ANN classifier, a large number of training data are required. Moreover, different pavement materials (such as the pavement structure in Table 1) have different properties; therefore, training different ANN models for different materials is necessary. More projects and materials will be included in our future studies.

\section{Data Availability}

The data required to reproduce these findings cannot be shared at this time as the data also forms part of an ongoing study.

\section{Conflicts of Interest}

The authors declare that there are no conflicts of interest regarding the publication of this paper.

\section{Acknowledgments}

This work was supported by Science and Technology Plan of Shandong Transportation Department (Grant no. 2019B48).

\section{References}

[1] O. Jeongho, "A review on intelligent compaction techniques in railroad construction," International Journal of Railway, vol. 7, no. 3, pp. 80-84, 2014.
[2] D. Liu, Y. Wang, J. Chen, and Y. Zhang, "Intelligent compaction practice and development: a bibliometric analysis," Engineering, Construction and Architectural Management, vol. 27, no. 5, p. 1213, 2019.

[3] M. Mooney and D. Adam, "Vibratory roller integrated measurement of earthwork compaction: an overview," in Proceedings of the 7th International Symposium on Field Measurements in Geomechanics, Boston, MA, USA, 2007.

[4] R. Anderegg and K. Kaufmann, "Intelligent compaction with vibratory rollers: feedback control systems in automatic compaction and compaction control," Transportation Research Record: Journal of the Transportation Research Board, vol. 1868, no. 1, pp. 124-134, 2004.

[5] R. Anderegg, D. A. von Felten, and K. Kaufmann, "Compaction monitoring using intelligent soil compactors," in Proceedings of the GeoCongress 2006: Geotechnical Engineering in the Information Technology Age, Atlanta, GA, USA, 2006.

[6] X. Zhu, S. Bai, G. Xue et al., "Assessment of compaction quality of multi-layer pavement structure based on intelligent compaction technology," Construction and Building Materials, vol. 161, pp. 316-329, 2018.

[7] D. J. White, P. K. R. Vennapusa, and H. H. Gieselman, "Field assessment and specification review for roller-integrated compaction monitoring technologies," Advances in Civil Engineering, vol. 2011, Article ID 783836, 15 pages, 2011.

[8] C. Wersäll, I. Nordfelt, and S. Larsson, "Soil compaction by vibratory roller with variable frequency," Géotechnique, vol. 67, no. 3, pp. 272-278, 2017.

[9] M. A. Mooney and R. V. Rinehart, "In situ soil response to vibratory loading and its relationship to roller-measured soil stiffness," Journal of Geotechnical and Geoenvironmental Engineering, vol. 135, no. 8, pp. 1022-1031, 2009.

[10] M. A. Mooney and R. V. Rinehart, "Field monitoring of roller vibration during compaction of subgrade soil," Journal of Geotechnical and Geoenvironmental Engineering, vol. 133, no. 3, pp. 257-265, 2007.

[11] M. Mazari, J. Beltran, R. Aldouri, and S. Nazarian, "Variability of intelligent compaction data on embankment and subgrade geomaterials," in Proceedings of the Geotechnical Frontiers 2017, Orlando, FL, USA, 2017.

[12] E. Reynders, "System identification methods for (operational) modal analysis: review and comparison," Archives of Computational Methods in Engineering, vol. 19, no. 1, pp. 51-124, 2012.

[13] B. Li and A. Der Kiureghian, "Operational modal identification using variational Bayes," Mechanical Systems and Signal Processing, vol. 88, pp. 377-398, 2017.

[14] K. H. Hsieh, M. W. Halling, and P. J. Barr, "Overview of vibrational structural health monitoring with representative case studies," Journal of Bridge Engineering, vol. 11, no. 6, pp. 707-715, 2006.

[15] G. F. Sirca Jr. and H. Adeli, "System identification in structural engineering," Scientia Iranica, vol. 19, no. 6, pp. 1355-1364, 2012.

[16] C. Devriendt, G. De Sitter, S. Vanlanduit, and P. Guillaume, "Operational modal analysis in the presence of harmonic excitations by the use of transmissibility measurements," Mechanical Systems and Signal Processing, vol. 23, no. 3, pp. 621-635, 2009.

[17] M. Barman, S. A. Imran, M. Nazari, S. Commuri, and M. Zaman, "Use of intelligent compaction in detecting and remediating under-compacted spots during compaction of asphalt layers," in Proceedings of the 2018 Civil Infrastructures 
Confronting Severe Weathers and Climate Changes Conference, Hangzhou, China, 2018.

[18] S. A. Imran, M. Barman, S. Commuri et al., "Artificial neural network-based intelligent compaction analyzer for real-time estimation of subgrade quality," International Journal of Geomechanics, vol. 18, no. 6, Article ID 04018048, 2018.

[19] M. Barman, M. Nazari, S. A. Imran et al., "Quality control of subgrade soil using intelligent compaction," Innovative Infrastructure Solutions, vol. 1, no. 23, 2016.

[20] Q. Zhang, T. Liu, Z. Zhang, Z. Huangfu, Q. Li, and Z. An, "Compaction quality assessment of rockfill materials using roller-integrated acoustic wave detection technique," Automation in Construction, vol. 97, pp. 110-121, 2019.

[21] Z. An, T. Liu, Z. Zhang et al., "Dynamic optimization of compaction process for rockfill materials," Automation in Construction, vol. 110, Article ID 103038, 2020.

[22] P. Dighe, A. Asaei, and H. Bourlard, "Sparse modeling of neural network posterior probabilities for exemplar-based speech recognition," Speech Communication, vol. 76, pp. 230-244, 2016.

[23] R. V. Darekar and A. P. Dhande, "Emotion recognition from Marathi speech database using adaptive artificial neural network," Biologically Inspired Cognitive Architectures, vol. 23, pp. 35-42, 2018.

[24] S. Mirsamadi and J. H. L. Hansen, "Multi-domain adversarial training of neural network acoustic models for distant speech recognition," Speech Communication, vol. 106, pp. 21-30, 2019.

[25] D. Palaz, M. Magimai-Doss, and R. Collobert, "End-to-end acoustic modeling using convolutional neural networks for HMM-based automatic speech recognition," Speech Communication, vol. 108, pp. 15-32, 2019.

[26] M. Zhan, M. Diao, L. Gao et al., "Neural networks for radar waveform recognition," Symmetry, vol. 9, no. 5, p. 75, 2017.

[27] M. F. Møller, "A scaled conjugate gradient algorithm for fast supervised learning," Neural Networks, vol. 6, no. 4, pp. 525-533, 1993. 